\title{
AN AUTONOMOUS SYSTEM FOR LINEAR AND ANGULAR MEASUREMENTS IN BIG SURFACES
}

\author{
B. Valera, R. Nava \& E. Miranda.
}

Centre of Applied Sciences and Technological Development, UNAM

Apdo. Postal 70-186, Coyoacán, 04510, México, D.F.

Tel. 5622 8602, Ext. 122 Fax. 56228624

E-Mail: valerab@aleph.cinstrum.unam.mx

Received: April $22^{\text {nd }} 2002$ and accepted January $7^{\text {th }} 2003$

\begin{abstract}
At present, warehouse construction industry makes large vertical stores over small areas causing vertical designs of vehicles and structures. In this situation, the floor must have constant levelness and high flatness in order to achieve the nominal performance of the very narrow aisle lift trucks. With this in mind, in this paper we present a novel system for levelness and flatness measurement based on big surfaces shape measurement. The system has linear and angular optical measurement devices on a mobile robot with automatic drive based on an embedded microcontroller. Readings from the robot are sent to a host computer by means of a radio frequency link for future analysis of level and flatness in agreement with the E1155-87 ASM standard. In addition, this work presents a PC program that receives data from the robot and then sends the data dynamically to a spreadsheet. The complete travel of the vehicle over the measurement area can be made with high degree of automation, reporting remarkable advantages compared to those of a manual process.
\end{abstract}

KEYWORDS: Mobile Robot, Flat Floors, Flatness and Levelness Measurements, OLE Automation.

\section{INTRODUCTION}

Some special floors are made in order to support the displacement of very narrow lift trucks over randomly or fixed routes. For instance, these kinds of floors are used in large height vertical warehouses, which are constructed to keep items in the smallest possible area. In such case, the area intended for the traffic is very narrow and the floor should be very flat and straight to avoid the vehicle staggering and crashing with nearby structures. Therefore, it is necessary to evaluate the floor features prior to the beginning of the warehouse operation. This information is useful for two main purposes: to check if the floor accomplishes with the expected tolerances, and to correct construction faults. Therefore, the main aim is to prevent out time fixes that could outcome in human and material losses [1].

Figure 1 shows the development of a big surface measurement in a vertical warehouse. This process was made in a manual approach, without any automated device, just using inductive angle and displacement measurement instruments. The previous experience involves a huge manual work of data reading and processing. For this reason, the measurement of big surfaces forces the use of automated systems that performs those tedious tasks and takes advantage of the modern electronic and computation systems. 


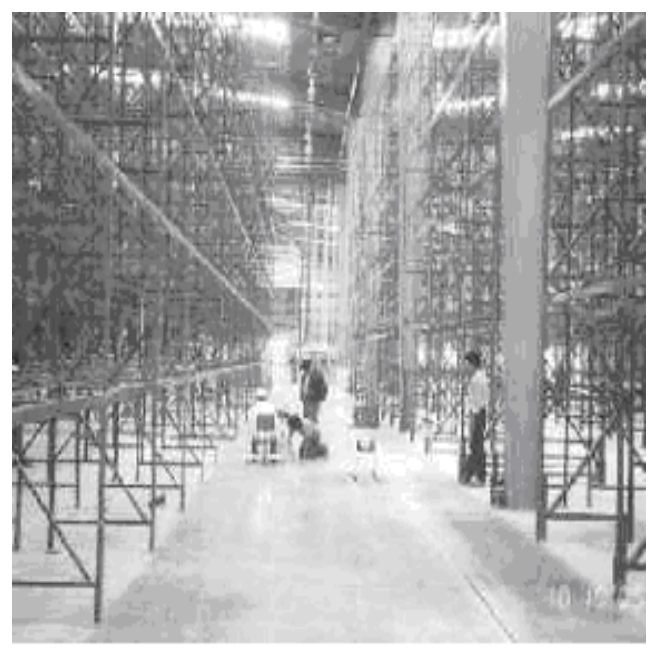

Figure 1. Floor measurement in a vertical warehouse. (Courtesy of A. D. Tec. Gerencia de Construcción, S. A. de C. V.)

The automated system described in this paper is used for big surfaces measurement, in order to assist or to certificate construction process in vertical warehouses. In our concept, the overall instrument is composed of a mobile robot used for data acquisition and the software that transports readings to a spreadsheet. However, the performance of the instrument is not limited to warehouses measuring; the range of applications could be extended to other types of big surfaces by means of implementing several measurement algorithms and some modifications to the spreadsheet.

Our system for automated linear and angular measurements constitutes an alternative approach to the little offer of similar instruments. With this implementation we are going in to in highly specialized services in an incipient industry.

The organization of this paper is as follows. Section two introduces the importance of evaluation of big areas. Section three points out the main system for linear and angular measurement. Section four shows the results of calibration process and some features reached.

\section{BIG SURFACES AND FLAT FLOORS}

Nowadays the construction industry is raising the storage capacity in warehouses by means of the incorporation of vertical designs in luggage compartments and lift trucks. Such approach increases the total amount of storage capacity about $60 \%$ or more, saving money with the use of already available space, as figure 2 shows. The key point then is to enlarge the warehouse height keeping the existing field. However, the floor that covers the field is also extensive, occupying thousands of square meters.

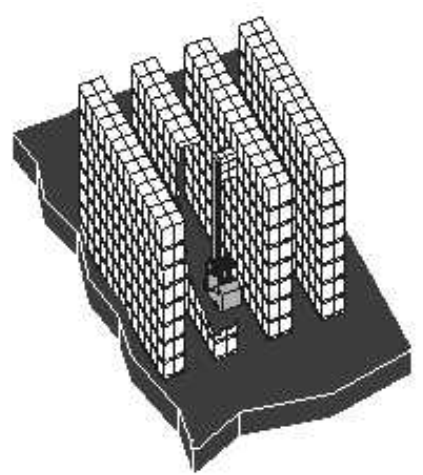

Figure 2. Vertical warehouse and lift truck 
Face Company first introduced the superflat term in the 70's to describe the levelness and flatness constraint in order to accomplish with the very narrow aisle (VNA) lift trucks terms. Those terms ensures the nominal speed without problems of VNA lift trucks moving over a traffic floor. Then, it is necessary to obtain high degrees in floor flatness and levelness to get high assurance in the VNA lift truck performance. For this reason the floor shape must be carefully measured. In addition, specialized maintaining techniques and a quality control program are also required both through the lifetime operation of the floor.

As a result, floors are measured concerning flatness and levelness by means of lineal and angular measurement devices. In this sense, the terms $F_{F}$ and $F_{L}$ qualifies the Floor Flatness and the Floor Levelness respectively. The literature reports the method used to obtain $F_{F}$ and $F_{L}$ numbers starting with dimensional measurements. For traffic floors, such as warehouse floors, the quality is given by

$$
F=\frac{100 F_{F}}{50 F_{L}}
$$

Then a superflat floor accomplishes with the Fnumber restriction of $₹>100$. Fnumbers are described below.

\subsection{Geometrical meaning of $F_{F}$}

Figure 3 shows $P_{0}, P_{1}$ and $P_{2}$ points that are separated at a distance of $304.8 \mathrm{~mm}\left(12^{\prime \prime}\right)$ between them on a imaginary straight line above the floor. Highest $C_{\max }$ is equal to $d_{1}+d_{2}$ and it can be expressed as a $F_{F}$ function like:

$$
C_{\max }=\frac{4.57}{F_{F}}[\mathrm{in}]
$$

or in millimeters:

$$
C_{\max }=\frac{116.08}{F_{F}}[\mathrm{~mm}]
$$

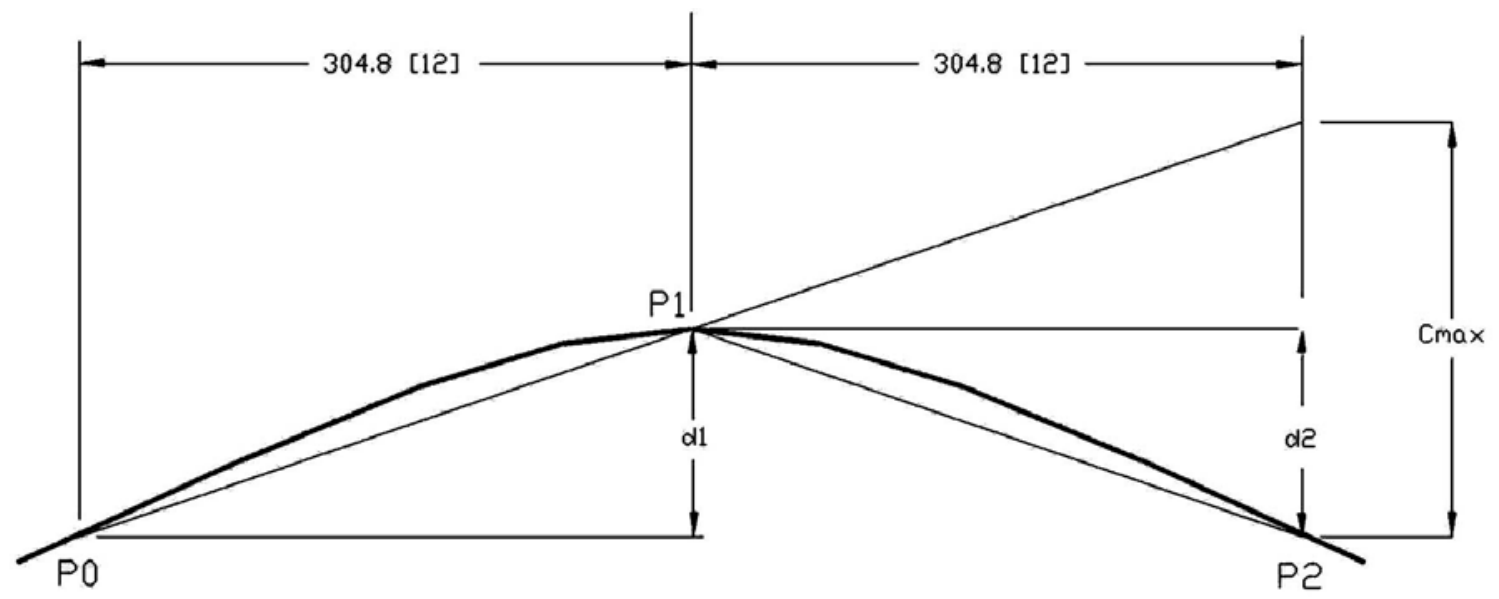

Figure 3. Geometrical meaning of $F_{F}$ 
$C_{\text {máx }}$ is the maximum permitted rate for certain quality floor, which depends on the $F_{F}$ number. Table 1 gives recommended values for $F_{F}$ numbers. By the way, for superflat floor $F_{F}=100$ then $C_{\text {máx }}=1.1608 \mathrm{~mm}\left(C_{\text {máx }}=0.0457^{\prime \prime}\right)$.

\subsection{Geometrical meaning of $F_{L}$}

$F_{L}$ is a number associated to the floor surface inclination. Figure 4 shows the maximal high difference $d_{\max }$ between $\mathrm{P}_{0}$ and $\mathrm{P}_{10}$ points, which are separately $3.048 \mathrm{~m}$ (10 ft). Highest $d_{\max }$ is equal to $h_{10}-h_{0}$ and it can be expressed as a $F_{L}$ function like:

$$
d_{\max }=\frac{12.5}{F_{L}}[\mathrm{in}]
$$

or in millimeters

$$
d \max =\frac{317.5}{F_{L}}[\mathrm{~mm}]
$$

Table I. Classification of concrete floors with its flatness and levelness, $F_{\digamma}$ and $F_{\iota}$ numbers.

\begin{tabular}{|c|c|c|c|c|}
\hline Floor classification & $\begin{array}{c}\text { Flatness and } \\
\text { levelness } \\
\text { requirements }\end{array}$ & $\begin{array}{c}F_{f} \\
\text { recommended }\end{array}$ & $\begin{array}{c}F_{l} \\
\text { recommended }\end{array}$ & $\begin{array}{c}\text { Tolerance Grade }(\mathrm{t}) \\
\text { recommended } \\
\mathrm{mm} \quad[\mathrm{in}]\end{array}$ \\
\hline $\mathrm{NC}$ & non critical & 18 & 10 & $\pm 25.4 \quad[1]$ \\
\hline $\mathrm{AV}$ & average & 25 & 18 & $\pm 19.05[3 / 4]$ \\
\hline BA & Better average & 35 & 25 & $\pm 12.7 \quad[1 / 2]$ \\
\hline VF & Very flat & 50 & 35 & $\pm 9.53[3 / 8]$ \\
\hline SF & Super flat & 100 & 70 & $\pm 6.35[1 / 4]$ \\
\hline
\end{tabular}

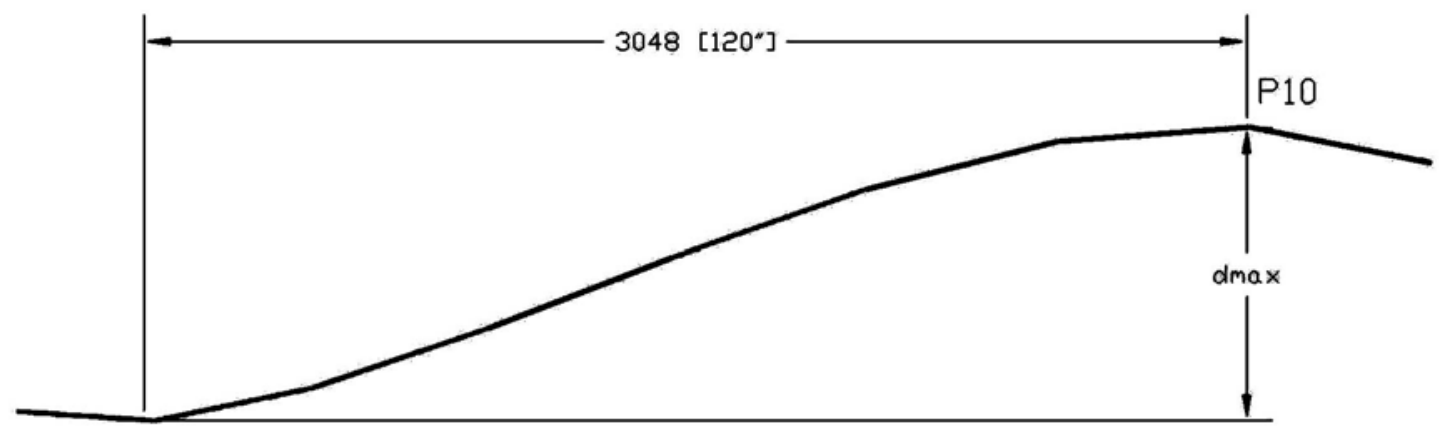

PO

Figure 4. Geometrical meaning of $F_{F}$

The concrete construction industry uses specific measurement instruments for particular kind of situations, i.e. roads or floors. An example is the profile, developed first by Face Company.

\section{SYSTEM DESCRIPTION}

In the measurement system there are two components: hardware and software, physically separated but interacting through a RF communications link. The first component is a mobile robot controlled automatically in a straight route 
on the floor under inspection and which transports optical devices for length and angle measurements. The same robot obtains and sends readings via RF to a host computer. The self-directed displacement of the robot is achieved by means of a laser-pointing device placed in the opposed end of the trajectory to cross. The robot is equipped with optical guidance sensors used to detect the deviations on the predefined trajectory so that the direction can be corrected. Also, the robot has sensors of length displacement that indicate the moment to acquire readings. The second component is a PC program and a receiving RF station awaiting the asynchronous arrival of readings to be processed to obtain the F-numbers that describe the quality of the floor. See scheme that describes the measurement system in figure 5.

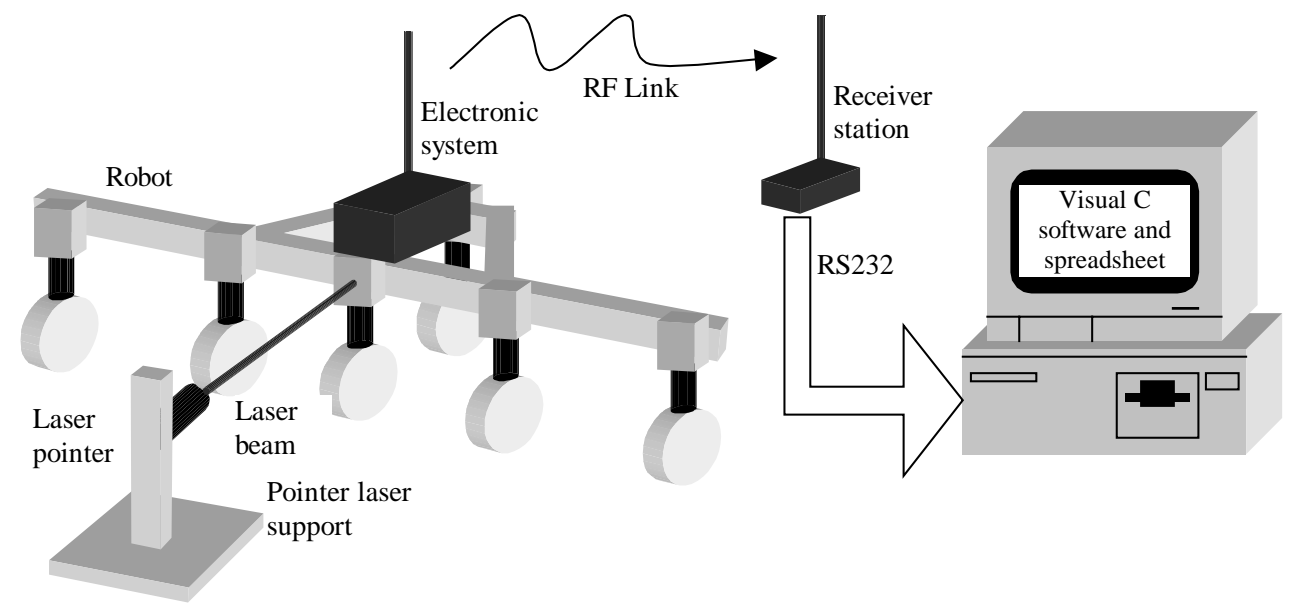

Figure 5. Autonomous system for linear and angular measurements in big surfaces

\subsection{Electronic stage}

The robot's electronic system is made up of a main control based on MC68HC11 microcontroller, and subsystems of guidance, self-governing displacement, data acquisition and RF communications. The program in assembler language for the HC11 implements the control algorithm in a strongly asynchronous interruption based approach. Figure 6 shows the electronic system. Refer to Figure 6 in the following description for the notation and details.

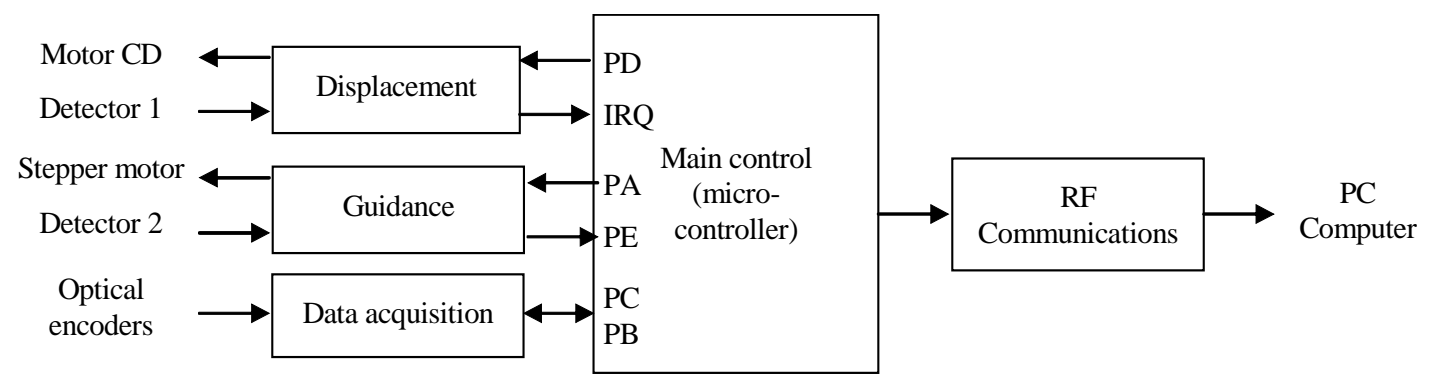

Figure 6. Electronic system

The self-governing displacement subsystem provides the electrical energy for the autonomous displacement of the robot with controlled mobility. The optical sensor identified as "Detector 1" is a simple opto-coupler device that senses the displacement like an odometer. The main wheel in the robot has a fixed index that is used to interrupt the light transmission in the transmitter-receiver pair at the opto-coupler device. This interruption generates a control signal to the main control. Detector 1 allows the controlled advance of the robot on the defined trajectory to a stable stop stable each $30 \mathrm{~cm}$ to capture readings of displacement and angle. Detector 1 generates an interruption, IRQ, in the microcontroller to look out the flow of the control algorithm. The movement of the robot is controlled by impelling a DC motor shared with the advance mechanism in the robot through port D of the HC11. 
The guidance subsystem stands for small deviations from the straight route and then corrects the direction of the mobile robot. The route is predefined by means of a laser pointer placed in the opposite side. The route deviation is sensed using an optical infrared sensor array denoted as "Detector 2" that is connected to the HC11 port E. The left displacement of the robot is sensed by a photo-transistor connected to pin PE7 and the right displacement is sensed by a photo-transistor connected to pin PE6. The control algorithm is fed with information from Detector 2 in the following form: if $\mathrm{PE} 7=1$ then the correction is to the right, if $\mathrm{PE} 6=1$ then the correction is to the left, if $\mathrm{PE} 7=\mathrm{PE} 6=0$ then there are no corrections. The correction is carried out by driving a stepper motor shared with to the direction mechanism in the robot. The stepper motor is governed through port A of the HC11.

The data acquisition subsystem consists of three interface circuits between microprocessor and optical encoders, LS7266R1 [2], three linear optical encoders for displacement measurement [3] and two optical pendulum encoders or inclinometers for angle measurement [4]. The optical encoders are displacement and angle transducers that are connected to a main digital controller by means of a LS7266R1 interface circuit. In this sense, the displacement and angle data is fed to the measurement algorithm. The HC11 controls the actions of configuration, reading and writing in the three LS7266R1 for the data acquisition through its ports C and B. The use of solid-state transducers implies a low consumption of power, which prolongs the duration of the batteries.

The RF communications subsystem consists of a transmitting module, TXM-900-HP-II [5], controlled by the RS232 port D of the HC11. The TXM altogether with the RXM, works as wireless replacement of the serial interface between two communications equipment. The TXM operates with OOK modulation in the $900 \mathrm{MHz}$ band, reaching distances up to $100 \mathrm{~m}$ in line of sight. The wireless link allows the independent operation of the robot in relatively long distances.

The RF communications subsystem is complemented with the receiving station in the opposed end of the link. A receiving module, RXM-900-HP-II [6], and a level shifter, MAX232, compose the receiving station. The RXM generates the received data signal and the MAX232 prepares the signal for the RS232 serial port of the computer.

The main control system is constituted by the MC68HC811E2 microcontroller [7], which is the brain of this measurement instrument. The MC68HC11 is an 8-bit MCU that uses HCMOS technology for small size and fast operation with a low consumption of energy and discharge immunity to the noise.

\subsection{Mechanical design}

Figure 7 shows five equidistant contact points: $A, B, C, D$ and $\mathrm{E}$. Points $B$ and $D$ are rigid supports on the surface in the measurement process which, for effect of readings processing, defines a straight line segment with an inclination $\alpha$ respect to the horizontal. The vertical elevations $a, c$ and e are deviations of the $A, C$ and $E$ points with respect to straight line $B D$.

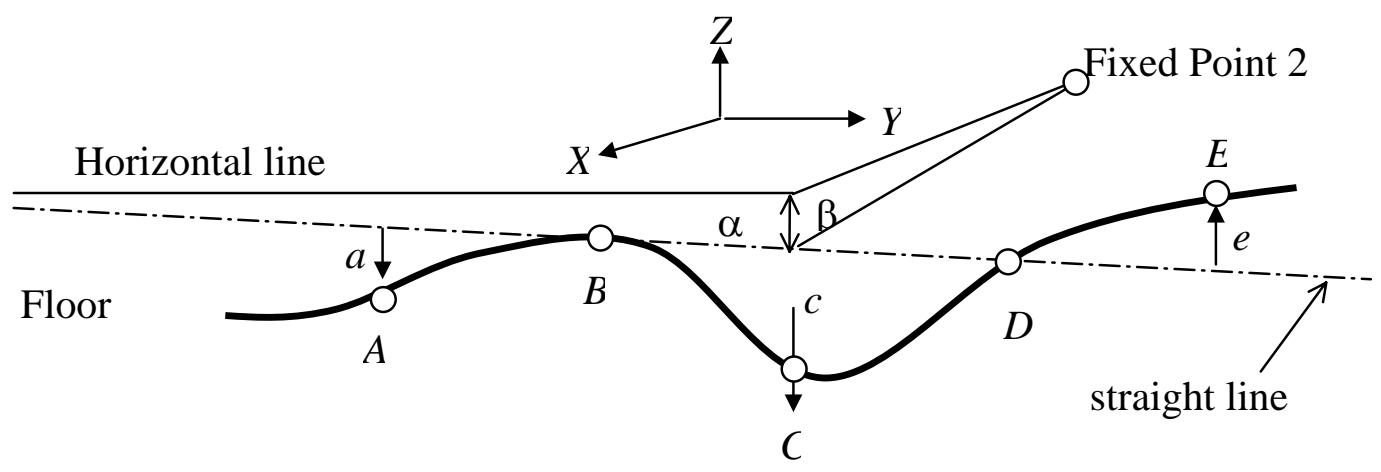

Figure 7. Measurement principle 
The elementary line straightness of the surface is obtained with these elevation data. Actually, the total topography of the surface is obtained by making the measurements of straightness of all the elementary lines previously defined of a surface and correlating the position between contiguous elementary lines.

The correlation of elementary lines becomes through the change of elevation of the point of contact with respect to the previous position in each measurement station. This change of elevation is defined by an angle $\beta$.

The values of angles $\alpha$ and $\beta$ as well as the values of deviation from straightness $A$ and $D$ of the straight line that crosses through supports $B$ and $C$ allows to describe the surfaces in agreement to the norms established for all the categories of floors as well as those established for all the categories of flat tables [8].

Figure 8 shows a CAD drawing of a mechanical structure with 3 fixed support points, 3 free-displacement points for deviation registration, $X Y$ angle registration and a support base for propose of calibration.

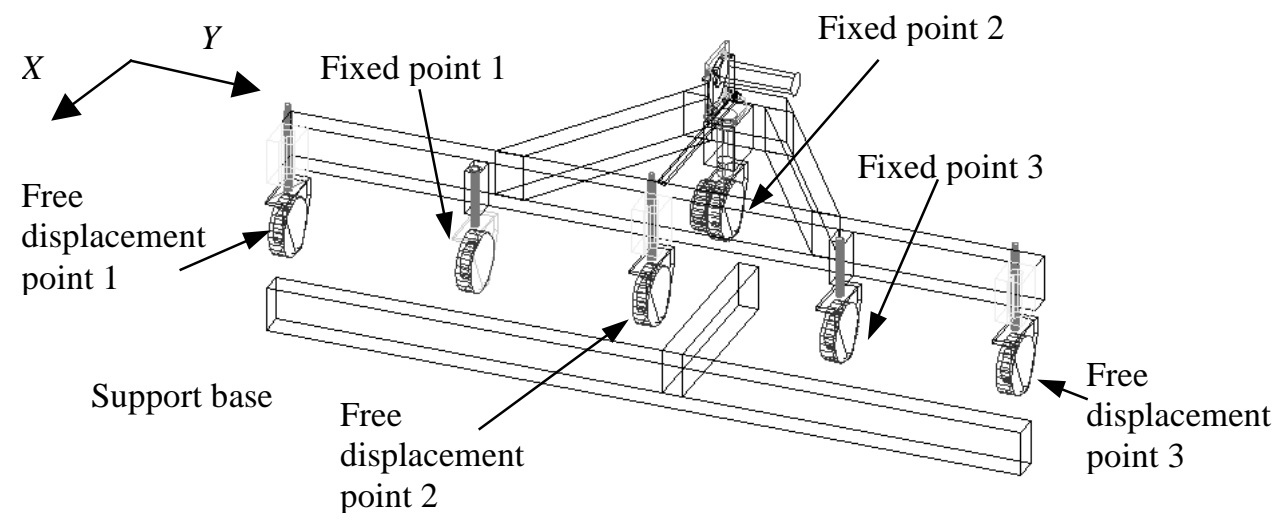

Figure 8. Mechanical design

\subsection{User interface software}

The user interface software allows to obtain the readings of the movable robot through a personal computer. In the scheme of figure 9 the program to access the serial port, Contro/Com, receives data in asynchronous form and dynamically sends them to a spreadsheet in Excel. The ControlCom program was specifically developed for this application using Visual $\mathrm{C}++6$ as a development tool. The dynamic exchange of asynchronous data, well known as OLE Automation, allows the acquisition of readings without the operator intervention [9]. Finally once the overall readings of a segment of the floor under inspection has been captured, the user can store the data, visualize it or calculate the $F$-numbers that describe the quality of the floor.

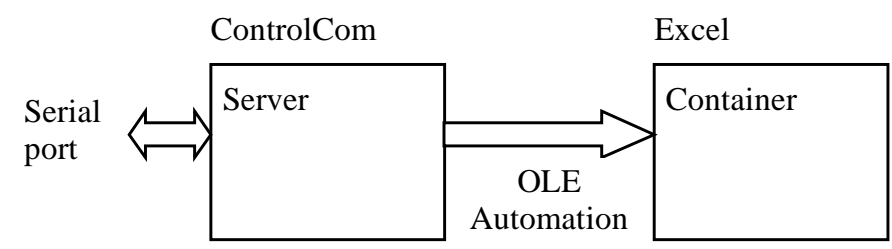

Figure 9. User interface software in a OLE Automation approach 


\section{RESULTS}

The results of the instrument can subjectively be evaluated when observing their performance during the measurement. However, the instrument as a whole can qualitatively be evaluated through the calibration of the displacement and angle measurement devices.

\subsection{Flatness and levelness measurement robot}

A robot for flatness and levelness measurement of big surfaces was developed; it is shown in figure 10. Their main functions provide the instrument with the following characteristics:

- $\quad$ Low power consumption. It requires dual supply of 5V-1.5W and 12V-8.4W, approximately

- $\quad$ Range of 20mm and 360arc-deg, covering two strips with $100 \mathrm{~m} \times 30 \mathrm{~cm}$.

- $\quad$ Resolution of 0,05mm and 0,05arc-deg.

- $\quad$ Accuracy of \pm 1 digit

- $\quad$ Self-displacement.

- $\quad$ Displacement speed of $0,3 \mathrm{~m} / \mathrm{s}$.

- Control algorithm implemented in software.

- $\quad$ Adjustments and calibration by software.

- Wireless and automatic transmission of linear displacement and inclination readings.

- Minimum intervention from the operator. Human intervention is required only for turning it on/off.

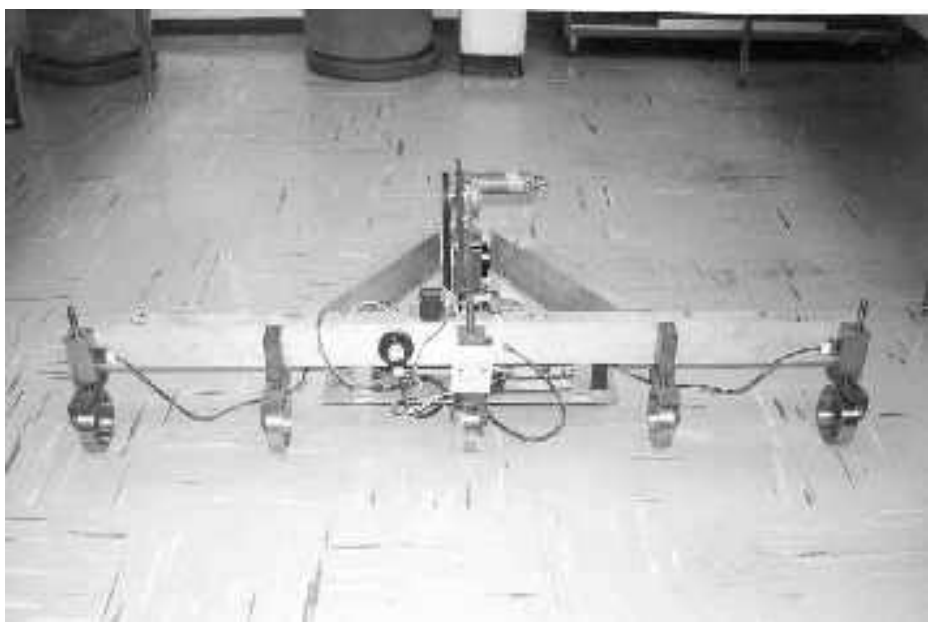

Figure 10. Flatness and levelness measurement robot

\subsection{User interface software}

A specific software was developed with application in the measurement of flatness and levelness using the OLE Automation technology that allows the dynamic data exchange between Windows 98/00 applications. The software, shown in figure 11, automates the process of capture of incoming readings without human intervention. Then, the developed software sends RS232 data of measurements to Excel for its processing. The main characteristics of software are the following ones:

- $\quad$ Automatic reception of asynchronous data in the serial port.

- $\quad$ Dynamic exchange of data with Excel (without any intervention from the operator).

- $\quad$ Friendly user interface under a Windows 98/00 platform.

- $\quad$ Object-Oriented Programming, OOP, which allows source code scalability and reusability. 


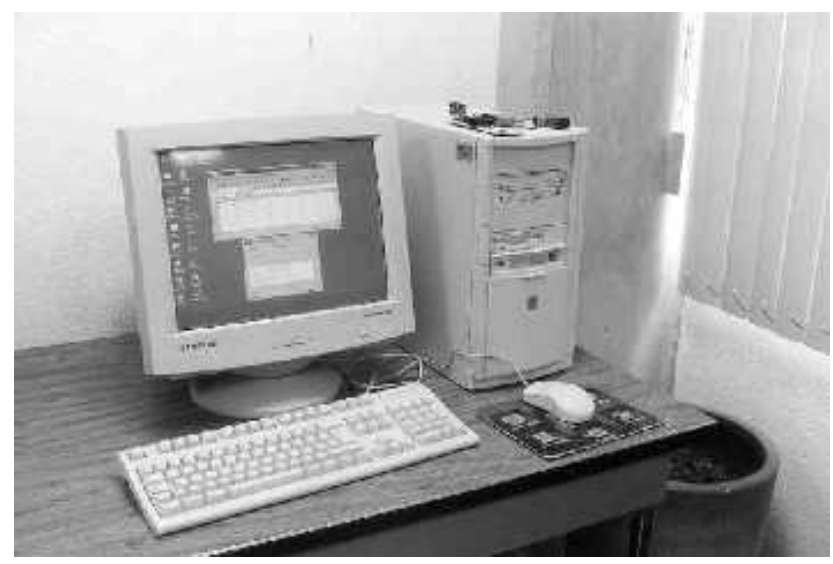

Figure 11. Host computer running the user interface software and acquiring data from RF receiver station

\subsection{Instrument calibration}

Two calibration experiments were developed in order to evaluate the performance of the measurement devices. The procedure for the linear calibration is shown in figure 12 and it consists in comparing master displacements in a Mitutoyo calibrator with those reported by the optical device in the robot.

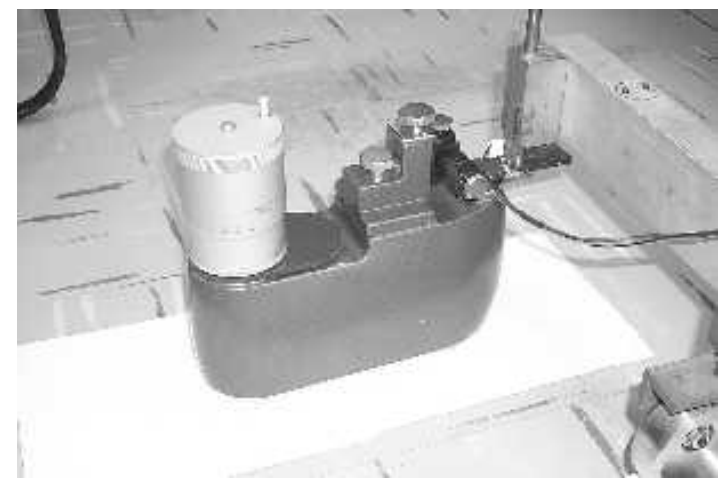

Figure 12. Linear calibration procedure

Figure 13 shows the angular calibration of the inclinometer device against a Nikon set of optical prism and autocollimator.

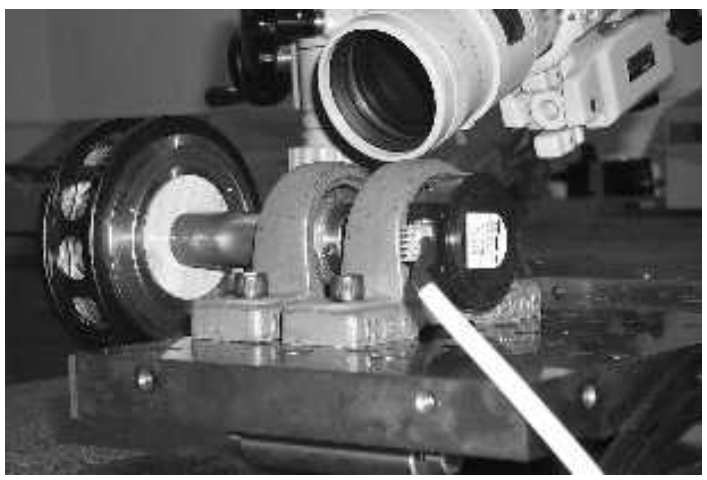

Figure 13. Angular calibration procedure 
The calibration charts of figures 14 and 15 were obtained from the experiments. The results allow us to appreciate the capacities of the instrument and to introduce corrections by software to improve the accuracy.

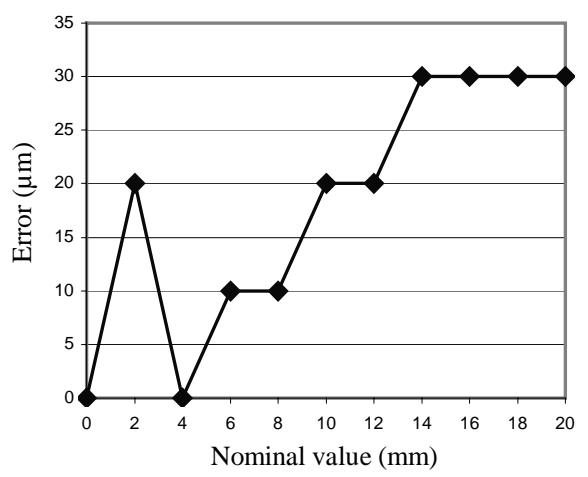

Figure 14. Linear calibration chart

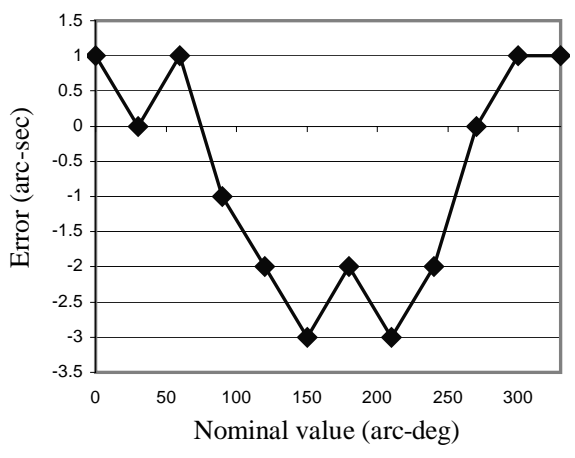

Figure 15. Angular calibration chart

\subsection{Measurement performance}

The performance in the measurement of a test surface confirms the expected results. Additionally, we can affirm the following:

- $\quad$ On the whole, the instrument is easy to operate. It only requires personnel with basic qualification in dimensional metrology and essential knowledge of the Windows environment.

- It provides an excellent comfort of operation. In this sense, the operator has the freedom to delegate the control of the measurement to the instrument and computer PC. In addition, the traverse range of the instrument $(100 \mathrm{~m})$ avoids that the operator accompanies the instrument on its route. The receiving station and computer PC remain fixed and the robot moves automatically in the surface under inspection.

\section{CONCLUSIONS}

Unlike the commercial equipment, our instrument requires a minimum operator intervention. On the whole, the instrument provides a high degree of automation in comparison to the commercial equipment available in the market.

The Metrology Laboratory of the Center of Applied Science and Technological Development, UNAM, is outfitted with the equipment and software to make measurements of flatness and levelness of big surfaces with a great quickness and almost null intervention from the user. Also it is possible to make use of these tools for the industry. Experience in 
the electronic development of recent semiconductor devices such as the mentioned RXM, TXM and R1 was acquired and assures the use of the instrument in the coming years. This allows us to approach projects of technological development with better technical capacity. As far as the ControlCom program concerns, it was verified that the automation of Microsoft Office software qualifies the programmers and users to develop and to use complex applications under a cooperative scheme of tasks.

\section{REFERENCES}

[1]. Face, The Face Companies Homepage, <http://faceco.com/compinfo.html>, January 2001.

[2]. US Digital, LS7266R1 Encoder to microprocessor interface chip, Technical data, US Digital Corp., Vancouver, USA, 1999, pp. 6.

[3]. US Digital, Optical encoder disk and linear strip, Technical data, US Digital Corp., Vancouver, USA, 1999, pp. 2.

[4]. US Digital, T2 Inclinometer, Technical data, US Digital Corp., Vancouver, USA, 1997, pp. 1.

[5]. Linx, High performance RF module TXM-900-HP-II, Linx Technologies, Oregon, USA, 1999, pp. 16.

[6]. Linx, High performance RF module RXM-900-HP-II, Linx Technologies, Oregon, USA, 1999, pp. 24.

[7]. Motorola, HC11 Reference manual, Motorola Inc., Phoenix, USA, 1991.

[8]. ACl Committee, Comentary on Standard Specifications for Tolerances for Concrete Construction and Materials (ACl 117-90), ACl Committee 117, pp. 7.

[9]. Kruglinsky D. J., Shepard G., Wingo S., Programming Microsoft Visual C++, Microsoft Press, USA, 1998, pp. 1153. 